\title{
Diversity of dendritic cells generated from umbilical cord or adult peripheral blood precursors
}

\author{
MAGDALENA SZARYŃSKA ${ }^{1}$, KRZYSZTOF PREIS ${ }^{2}$, PIOTR ZABUL ${ }^{3}$, ZBIGNIEW KMIEĆ $^{\prime}$ \\ ${ }^{1}$ Department of Histology, Faculty of Medicine, Medical University of Gdansk, Poland \\ ${ }^{2}$ Department of Obstetrics, Faculty of Medicine, Medical University of Gdansk, Poland \\ ${ }^{3}$ Department of Obstetrics \& Gynaecology, St. Wojciech Specialist Hospital, Independent Public Complex of Integrated Health Care Units \\ in Gdansk, Poland
}

\begin{abstract}
Following the discovery of methods to generate large numbers of specific dendritic cells (DCs) ex vivo, the possibility of exploiting these cells in immunotherapeutic strategies will become a reality. It seems to be rationally to analyse the influence of the precursor source for further features and applications.

For the needs of the given project DCs were derived from precursors derived from adult peripheral blood ( $A P B)$ and umbilical cord blood (UCB). During some expansions of $U C B C D 34^{+}$cells were separated giving non-adherent DCs (NA-DCs) or adherent DCs (A-DCs), whereas DCs derived from UCB precursors without separation gave rise to All-DCs. DC subpopulations were stimulated by lipopolysaccharides (LPS) or interferon- $\gamma($ IFN- $\gamma)$, and afterwards the morphology, phenotype, and stimulatory properties were analysed.

Our findings demonstrated that DCs generated from $A P B$ and $U C B$ precursors were not equivalent and exhibited opposite features when expanded in comparable conditions. Additionally, all three subpopulations of UCB-derived DCs presented functional dissimilarities. Based on our results we concluded that the precursor source and the composition of media must be considered as crucial to the success of potential therapeutic application.
\end{abstract}

Key words: dendritic cells, haematopoietic stem cells, monocytes.

(Centr Eur J Immunol 2018; 43 (3): 306-313)

\section{Introduction}

Among the mature blood cells, dendritic cells (DCs) are the most potent immune cells for antigen presentation and orchestration of the immune system. Traditionally these cells have been difficult to isolate due to their low concentration in peripheral blood (approximately $0.01 \%$ ) and the lack of a well-defined DC-specific marker. Following the discovery of methods to generate large numbers of DCs ex vivo, the possibility of exploiting these cells in a number of immunotherapeutic strategies has become a reality [1-3]. Mature DCs can be generated in vitro from the early haematopoietic $\mathrm{CD} 34^{+}$progenitors of cord blood (UCB), peripheral blood (APB), or bone marrow (BM) or their late monocytic precursors using a variety of cytokine combinations [1, 4-10]. Additionally, haematopoietic differentiation from induced pluripotent stem cells has been investigated recently [6]. Because, DC functions are effected by patients' (donor) health, cytokine selection for culture, and isolation procedures, DC precursors from all sources have been used in clinical trials depending on the goal [11].

When autologous cells cannot be taken into consideration as the source for DC generation because of some genetic defects, UCB haematopoietic stem cells (HSCs) give a great opportunity to expand DCs with specific features for patients with malignant and non-malignant diseases $[9,12]$. One of the advantages of the UCB is its relative accessibility as a source of HSCs, which can be cryopreserved for many years and ready to use [13]. Additionally, UCB HSCs display higher proliferative capacity and repopulating potential than BM HSCs. Moreover, they evoke weaker graft versus host $(\mathrm{GvH})$ reaction with the same human leukocyte antigen (HLA) matching in comparison to their BM counterparts [14, 15].

Comparison of $\mathrm{CD}_{3} 4^{+}$cell-derived DCs and monocyte-derived DCs from the same patient demonstrated similar morphology and performance in mixed lymphocyte reaction, while differences in some surface markers such as CD86 and HLA-DR were observed [3]. A difference in the

Correspondence: Magdalena Szaryńska, PhD, Department of Histology, Faculty of Medicine, Medical University of Gdansk, 1 Dębinki St., 80-210 Gdansk, Poland, e-mail: mszarynska@gumed.edu.pl

Submitted: 8.02.2017; Accepted: 24.04.2017 
capacity to activate $\mathrm{CD} 8^{+} \mathrm{T}$ cells was also reported in these two populations [16]. Scientists established optimised culture systems, which provided important advances for generating different subpopulations of DCs: antigen-presenting DCs (APC), suppressive DCs, or cytotoxic DCs [9, 17-19].

Although numerous studies have been published, we discovered some innovative differences between DC expanded from precursors isolated from UCB and APB. The most interesting were observations on UCB precursors, which during culture divided into two distinct subpopulations giving DCs with contrasting features. We investigated the phenotype and, when possible, stimulatory properties of DCs expanded from the mentioned precursors. In our study, monocyte-derived DCs were treated as a standard model for exploring DC properties.

\section{Material and methods}

\section{Dendritic cell culture from peripheral blood monocytes}

As peripheral blood samples we used leukocyte-platelet coats $(n=36)$ obtained from volunteers recruited during the routine medical consultation in the Regional Blood Bank in Gdansk, and only the healthy ones were included in this study. All subjects were informed about the study, and signed, written consent was approved by the Ethical Committee of the Medical University of Gdansk. APB mononuclear cells were separated by Histopaque ${ }^{\circledR}$-1077 (Sigma) gradient centrifugation at $1200 \mathrm{~g}$ for $30 \mathrm{~min}$ at room temperature. After isolation and erythrocyte lyses, the cells were washed and prepared for further isolation steps. To separate monocytes, PBMCs were cultured for $24 \mathrm{~h}$ on an adhesive Petri dish in RPMI1640 supplemented with FBS (10\%, PAA), L-glutamine (2 mM, Sigma), penicillin (100 U/ml, Sigma), and streptomycin $(100 \mu \mathrm{g} / \mathrm{ml}$, Sigma $)$, at $37^{\circ} \mathrm{C}, 5 \%$ $\mathrm{CO}_{2}$, and $95 \%$ humidity. After incubation time, medium containing non-adherent cells was gently removed and the plate with adherent cells was put on ice for 30 minutes. Afterwards, the monocyte layer was harvested using a scraper.

A total of $1 \times 10^{6}$ monocytes $/ 1 \mathrm{ml}$ were placed on 24-well plates in medium supplemented with GM-CSF (50 ng/ml, R\&D) and IL-4 (100 ng/ml, R\&D) for seven days. On day 3, half of the medium was replaced with fresh medium with cytokines. On day 6, cells were subjected to maturation with lipopolysaccharide (LPS) $(10 \mu \mathrm{g} / \mathrm{ml}$ or $100 \mu \mathrm{g} / \mathrm{ml}$, Sigma) or interferon- $\gamma(\mathrm{IFN}-\gamma)(5 \mathrm{ng} / \mathrm{ml}$ or $10 \mathrm{ng} / \mathrm{ml}, \mathrm{R} \& \mathrm{D})$ for 24 hours. Cell differentiation and maturation was monitored and documented by a light microscope equipped with a digital camera.

\section{Dendritic cell culture from $\mathrm{CD}^{+}{ }^{+}$cells from human umbilical cord blood}

UCB samples were collected to CPD-containing bags from 20 volunteers at the moment of delivery in the Depart- ment of Obstetrics of the Medical University of Gdansk, Poland. The neonates with major anomalies and with major delivery complications (including caesarean section) were excluded from the study. The procedure was conducted with parental permission in an aseptic manner by experienced hospital staff. The research was approved by the Ethics Committee of the Medical University of Gdansk. All UCB blood units were subject to procedure not later than $12 \mathrm{~h}$ after collection.

The EasySep ${ }^{\circledR}$ Human Cord Blood CD34 Positive Selection Kit (Stem Cell Technologies) is a two-step cell isolation kit. CD34+ cells were first pre-enriched from fresh whole cord blood using the RosetteSep® Human Cord Blood CD34 Pre-Enrichment Cocktail by negative selection designed to deplete granulocytes and RBCs. Following pre-enrichment, cells were positively selected using EasySep ${ }^{\circledR}$ Human CD34 Positive Selection Kit. RosetteSep ${ }^{\circledR}$ Human Cord Blood CD34 Pre-Enrichment Cocktail was added, and after 10 minutes of incubation the samples were diluted and layered on Histopaque ${ }^{\circledR}-1077$ (Sigma Aldrich). After centrifugation (1200 g, $30 \mathrm{~min}, \mathrm{RT}$ ) cells were subjected to magnetic positive selection. $2 \times 10^{8}$ mononuclear cells/ml were incubated with EasySep ${ }^{\circledR}$ Positive Selection Cocktail for 15 minutes and with EasySep ${ }^{\circledR}$ Magnetic Nanoparticles for next 10 minutes. Afterwards, the suspension with stained cells was placed in magnet and the steps: 10-minute incubation - pouring off supernatant suspension were repeated five times. After final centrifugation the positively selected CD $34^{+}$cells were ready for use.

$0.8 \times 10^{6}$ cells $/ \mathrm{ml}$ were placed on $24-w e l l$ plates in RPMI1640 supplemented with FBS (10\%, Sigma Aldrich), L-glutamine (2 mM, Sigma Aldrich), penicillin (100 U/ml, Sigma Aldrich), and streptomycin $(100 \mu \mathrm{g} / \mathrm{ml}$, Sigma Aldrich), GM-CSF (50 ng/ml, R\&D), IL-4 (50 ng/ $\mathrm{ml}, \mathrm{R} \& \mathrm{D})$, and $\mathrm{SCF}(50 \mathrm{ng} / \mathrm{ml}, \mathrm{R} \& \mathrm{D})$ at $37^{\circ} \mathrm{C}, 5 \% \mathrm{CO}_{2}$, $95 \%$ humidity for 17 days. On days 4,8 , and 12 half of the medium was replaced with fresh medium with cytokines. On the $16^{\text {th }}$ day cells were subjected to maturation with LPS (100 $\mu \mathrm{g} / \mathrm{ml}$, Sigma Aldrich) or IFN- $\gamma(10 \mathrm{ng} / \mathrm{ml}$, $R \& D)$ for 24 hours. During 10 cultures, on the $8^{\text {th }}$ day the non-adherent cells were removed and placed into a new well. These non-adherent precursors and remaining adherent cells were treated the same until the end of the culture, giving rise to, respectively, non-adherent DCs (NA-DCs) or adherent DCs (A-DCs). DCs derived from UCB CD34 precursors without separating into subpopulations gave rise to All-DCs. Cell differentiation and maturation was monitored and documented by a light microscope equipped with a digital camera.

\section{Cytometric analysis of UCB HSCs}

$\mathrm{CD} 34^{+} \mathrm{CD} 45^{\text {low }}$ haematopoietic stem cells were stained with the following cocktail of monoclonal antibodies purchased from BD Biosciences: anti-CD34-PE, anti-CD45-PerCP, and matched isotype controls. After 
30 min incubation in the dark, on-ice samples were fixed and prepared for further analysis. Flow cytometric analysis was performed using an LSRII flow cytometer (BD).

\section{Cytometric analysis of dendritic cells}

Differentiating cells during each passage and after stimulation were stained with the following cocktail of monoclonal antibodies purchased from BD Biosciences: anti-CD3-PE, anti-CD14-PerCP, anti-CD66b-PE, antiCD16-PerCP-Cy5.5, anti-CD11c-APC, anti-CD80-PE, anti-HLA-DR-PerCP, and matched isotype controls. After 30 min of incubation in the dark, on-ice samples were fixed and prepared for further analysis. Flow cytometric analysis was performed using an LSRII flow cytometer (BD). For death cell evaluation a 7AAD Via Probe (BD) was used. After adding $10 \mu \mathrm{m}$ of Via Probe samples were incubated for 30 minutes, washed, and resuspended in PBS prior to cytometric analysis.

\section{Mixed lymphocyte cultures (MLC)}

The stimulatory abilities of DCs derived from different precursors were measured during mixed leukocyte culture. Effectors were non-adherent mononuclear cells of peripheral blood or umbilical cord blood. Autologous $\gamma$-irradiated DCs modulated the response of effectors to $\mathrm{CD} 3$ / CD28 Dynabeads (Dynal) in a U-bottom 96-well plate and cultured for five days. Proliferation was measured after $3 \mathrm{H}$-thymidine $(0.5 \mu \mathrm{Ci} /$ well $)$ incorporation during incubation for the last $16 \mathrm{~h}$. Cells were harvested using a cell harvester and counted on a liquid scintillation counter (Cell Harvester, Scatron). Effectors were plated in a concentration of $5 \times 104$ cells per well in co-cultures with expanded DCs in different proportions (Effectors : DC $-2: 1,1: 1$, $1: 2,1: 4,1: 8$ ) and the following ratio mixtures: $1: 0$ and $0: 1$ as internal controls. The controls $S$ and $2 S$ contained effectors stimulated with either a single or double dose of anti-CD3/CD28 beads.

\section{Statistics}

Data were computed using the software Statistica 8.0 (Statsoft, Poland). The analysis of data obtained from the patients was based on non-parametric tests and results were analysed using: U Mann-Whitney test, Spearman rank correlation coefficients and ANOVA Kruskal-Wallis test. Values of $p<0.05$ were considered as statistically significant.

\section{Results}

Six-day cultures of APB monocytes yielded cells displaying fibroblast-like morphology with fine cytoplasmic processes. After 24-h stimulation with LPS cells became much longer, and the higher the concentration of LPS, the longer the processes that DCs exhibited (Fig. 1). After 24-h culture with IFN- $\gamma$, the vast majority of cells showed irregular cell forms and adhered to the dish, whereas the increased concentration of IFN- $\gamma$ did not influenced further cell morphology.

The culture of All-DCs from UCB CD $34^{+}$precursors provided cells with similar morphology to DCs derived from APB monocytes (Fig. 1). Surprising observations were made on these cells after they were divided into fractions - adhering (A) and non-adhering (NA). DCs derived from UCB precursors were likely to lose their adhesive properties during culture, and according to this phenomenon they were separated into adhering (giving rise to A-DCs) and non-adhering (giving rise to NA-DCs) precursors. A-DCs, NA-DCs, and All-DCs were expanded in vitro in the same conditions. The morphology of cells derived from these distinct types of precursors exhibited different morphology (Fig. 2). The A precursors presented strong adhesion to the dish for the whole culture time. After stimulation A-DCs acquired distinct forms, but still were adhering cells. LPS stimulated lengthening of cells with long cytoplasmic projections while IFN- $\gamma$ stimulated irregular widened forms (Fig. 2). NA precursors conserved floating spherical cell forms until the end of our analysis. Stimulation of immature NA-DCs increased the number and length of their cytoplasmic projections, and both LPS and IFN- $\gamma$ induced very similar changes.

Culture (of all experimental options) yielded valuable DCs, differentiation and maturation of which were confirmed by the presence of DC-specific markers: CD11c, CD80, and HLA-DR (Fig. 3, Fig. 4) and the absence of monocyte/macrophage and granulocytes - associated membranous proteins, respectively - CD14 and CD66b (data not shown). Increased concentrations of IL-4 and GM-CSF up to $100 \mathrm{ng} / \mathrm{ml}$ did not significantly change the morphology or specific marker expression on DCs (data not shown). The number of viable stimulated cells remained the same quantity as the control group as assessed with the usage of a 7AAD probe.

Because of the high diversity of the DC precursor population used for each expansion, the analysis of phenotypic changes of DCs after stimulation was based on the ratio between the proportion of DCs after stimulation in comparison to unstimulated control cells.

After IFN- $\gamma(10 \mathrm{ng} / \mathrm{ml})$ stimulation CD80 expression was greatly enhanced in APB monocyte-derived DCs and All-UCB precursor-derived DCs, whereas it was significantly decreased in NA-DCs and A-DCs, and this decline was greater for NA-DCs (Fig. 3, Fig. 4).

The influence of LPS on the analysed DC subpopulations was also tested. The results show that LPS increased the proportion of $\mathrm{CD} 80^{+} \mathrm{DC}$ derived from $\mathrm{APB}$ monocytes, All-UCB precursors and UCB NA precursors. Furthermore, LPS significantly decreased the level of CD80 ${ }^{+}$A-DCs in comparison to unstimulated control DCs (Fig. 3, Fig. 4). 

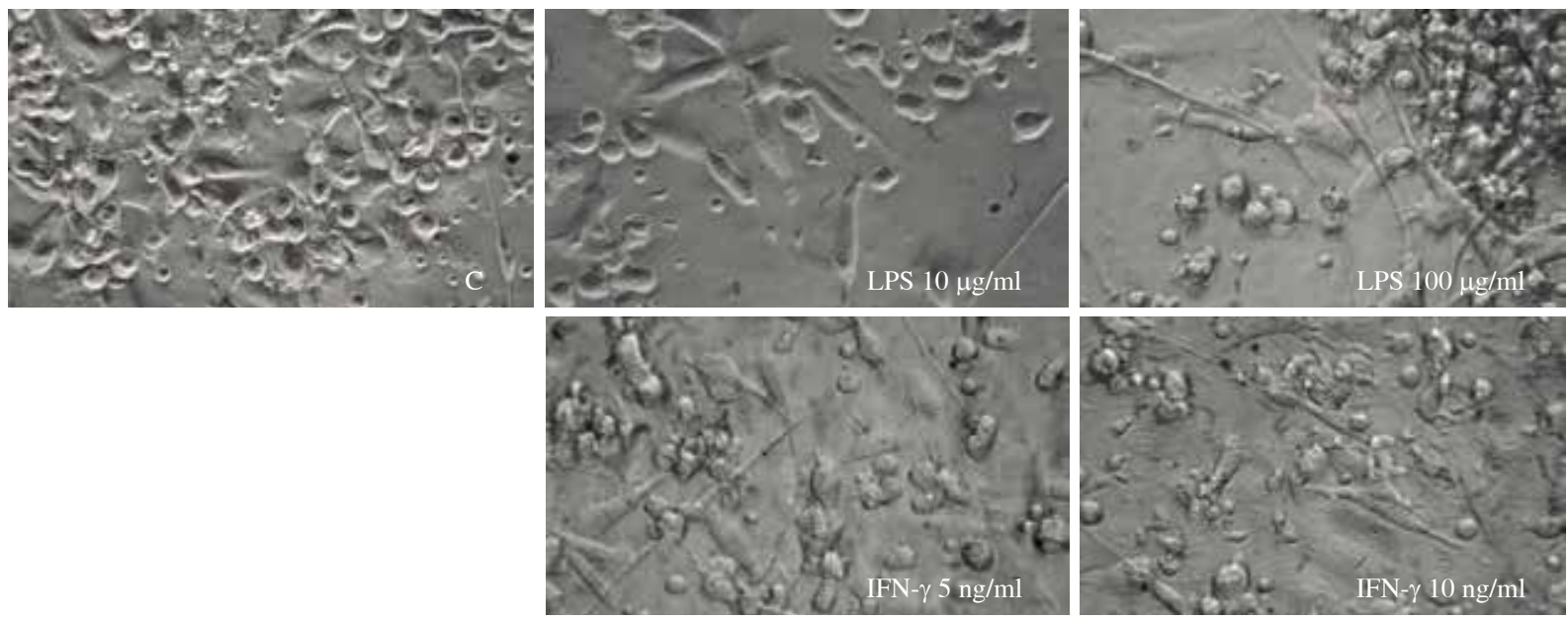

Fig. 1. The morphology of dendritic cells expanded from APB monocytes and stimulated with LPS $(10 \mu \mathrm{g} / \mathrm{ml}$ or $100 \mu \mathrm{g} / \mathrm{ml})$ or interferon- $\gamma(\mathrm{IFN}-\gamma)(5 \mathrm{ng} / \mathrm{ml}$ or $10 \mathrm{ng} / \mathrm{ml})$ in comparison to unstimulated control cells (C)
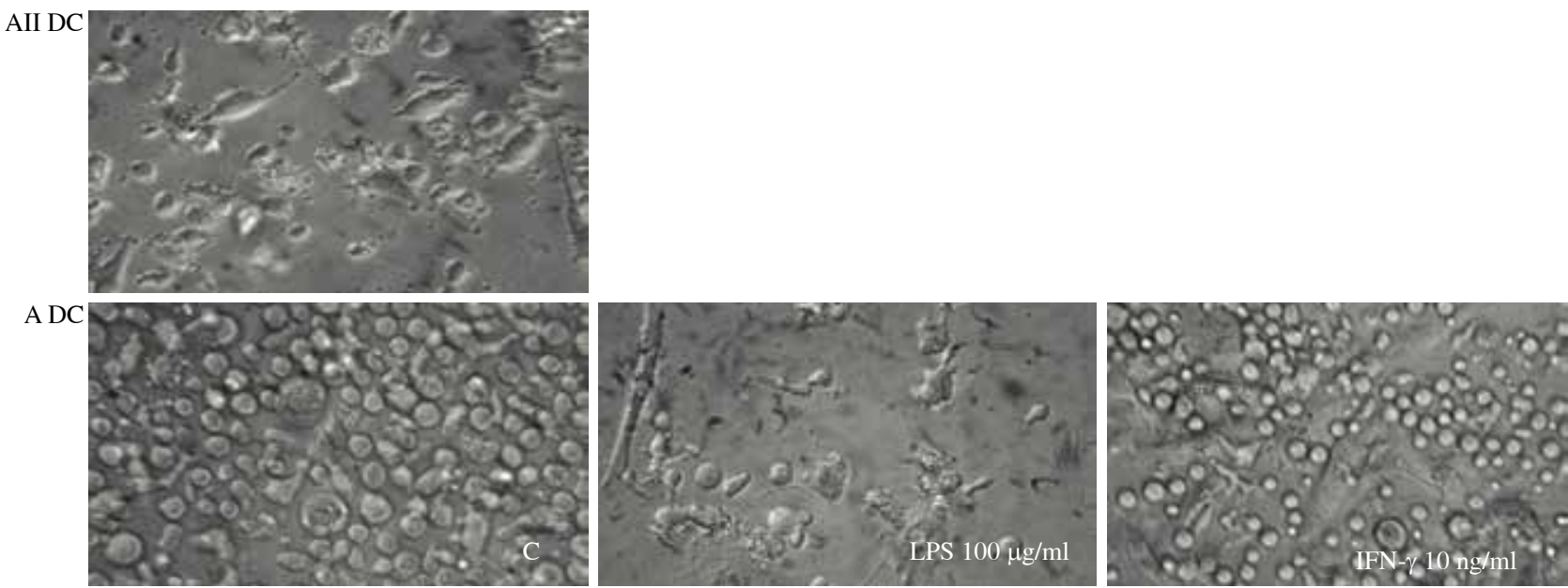

NA DC
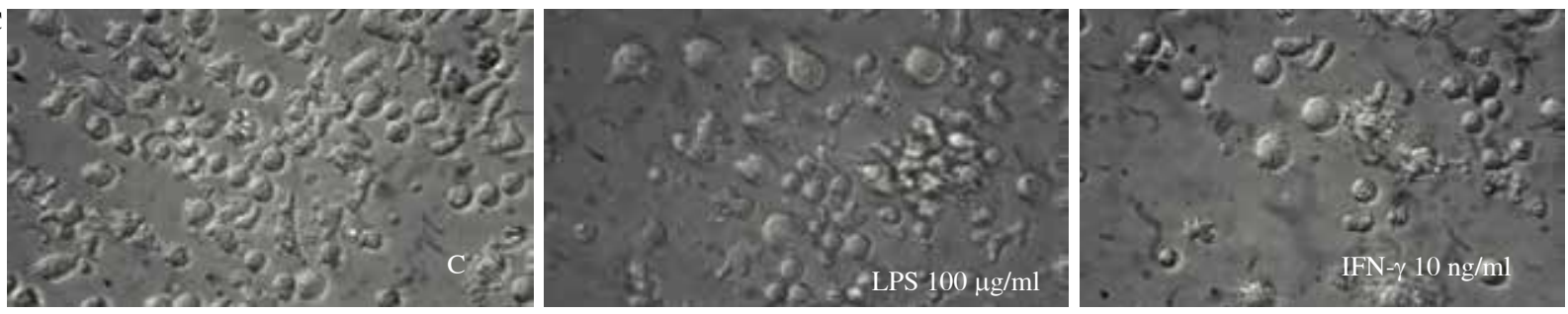

Fig. 2. The morphology of dendritic cells (DCs) expanded from UCB CD $34^{+}$precursors and stimulated with LPS $(100 \mu \mathrm{g} / \mathrm{ml})$ or IFN- $\gamma(10 \mathrm{ng} / \mathrm{ml})$ in comparison to unstimulated control cells $(\mathrm{C})$. UCB precursors were separated into adhering (giving rise to A-DCs) and non-adhering (giving rise to NA-DCs) fractions and cultured in the same conditions as All-CB precursors (giving rise to All-DCs)

The results obtained in MLC reactions showed that DCs generated from APB monocytes have the capacity to induce effector cell proliferation whereas All-DCs were unable to induce stimulatory response but were inhibitory due to decreasing the effector proliferation rate. $\mathrm{ABP}$ monocyte-derived DCs enhanced effector proliferation after LPS $(10 \mu \mathrm{g} / \mathrm{ml} ; 100 \mu \mathrm{g} / \mathrm{ml})$ and IFN- $\gamma(10 \mathrm{ng} / \mathrm{ml})$ stimulation in comparison to unstimulated control cells. The higher the concentration of LPS was used, the higher the number of CD80+ DCs found; additionally, the higher the concen- 

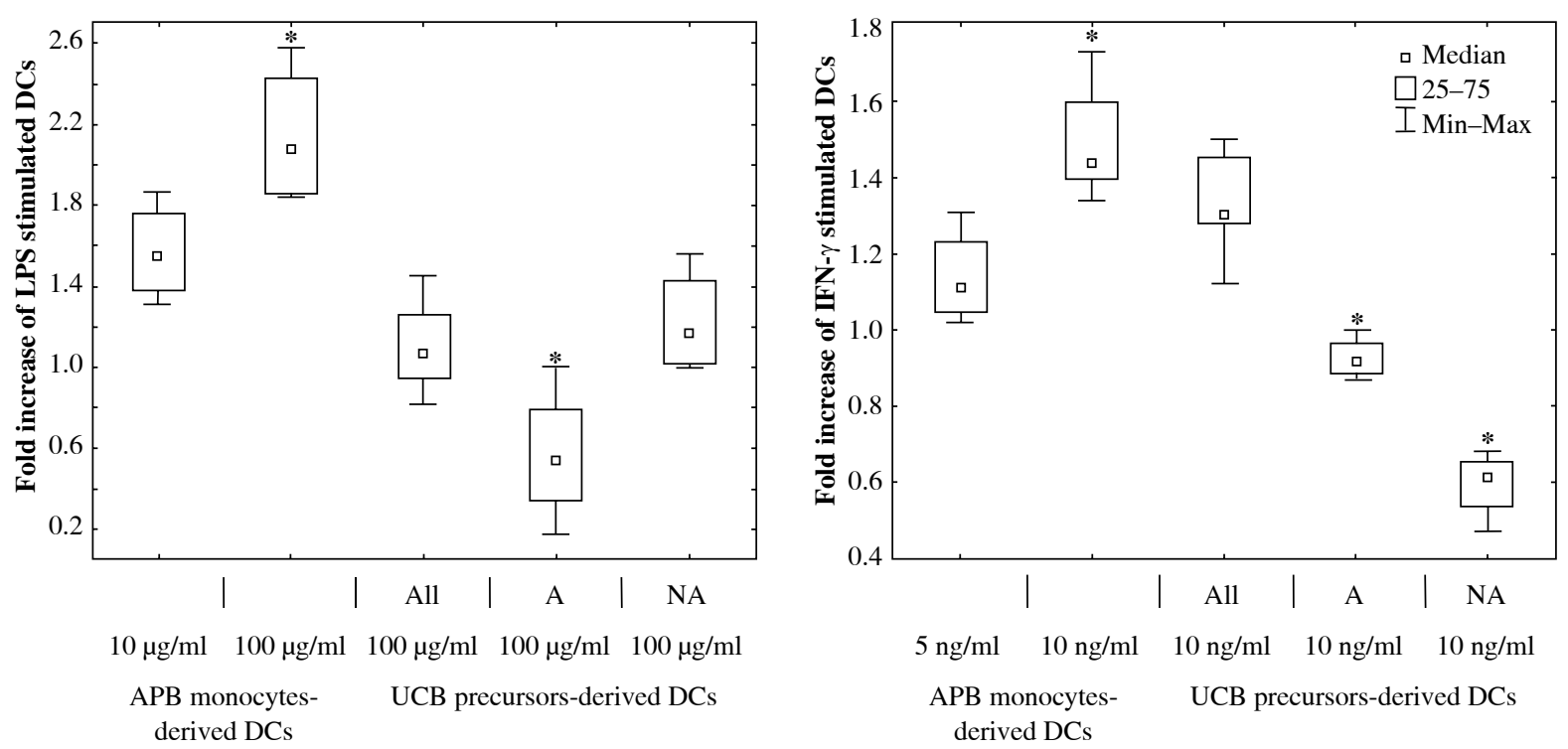

Fig. 3. The comparison of effect of LPS $(10 \mu \mathrm{g} / \mathrm{ml}$ or $100 \mu \mathrm{g} / \mathrm{ml})$ and IFN- $\gamma(5 \mathrm{ng} / \mathrm{ml}$ or $10 \mathrm{ng} / \mathrm{ml})$ stimulation on DCs

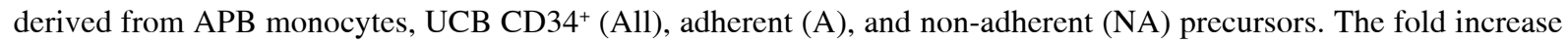
(based on the proportion of DCs measured with flow cytometry) of CD11c+HLA-DR+CD80+ DCs determined by comparing data with unstimulated control cells. The control value was fixed at the level of $1\left(^{*}-\right.$ statistical significance, ANOVA Kruskal-Wallis test, $p<0.05$ )
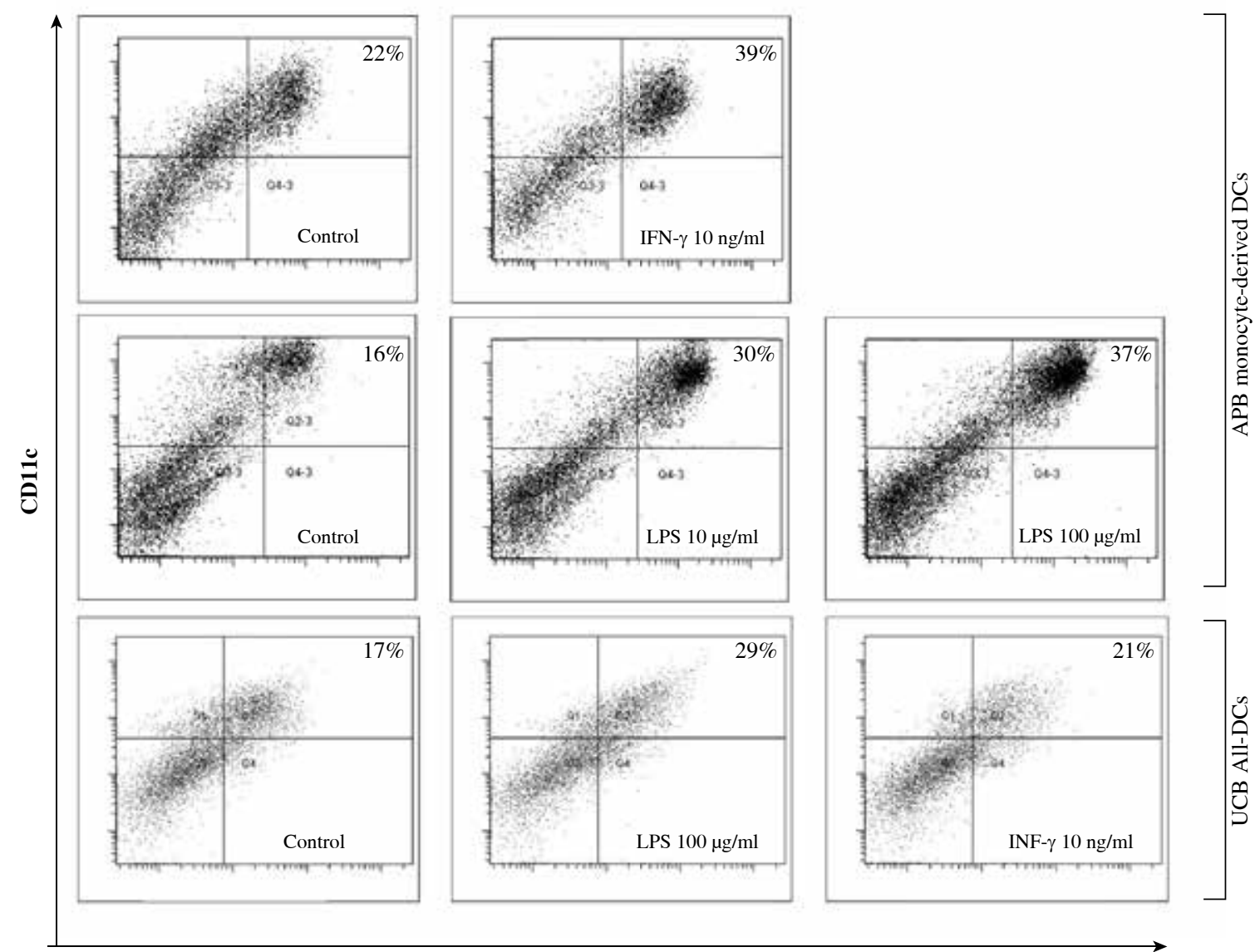

CD80

Fig. 4. Representative flow cytometric dot plots illustrating the proportion of CD11c+HLA-DR+CD80 $0^{+}$Cs derived from APB monocytes and UCB All-DC precursors 

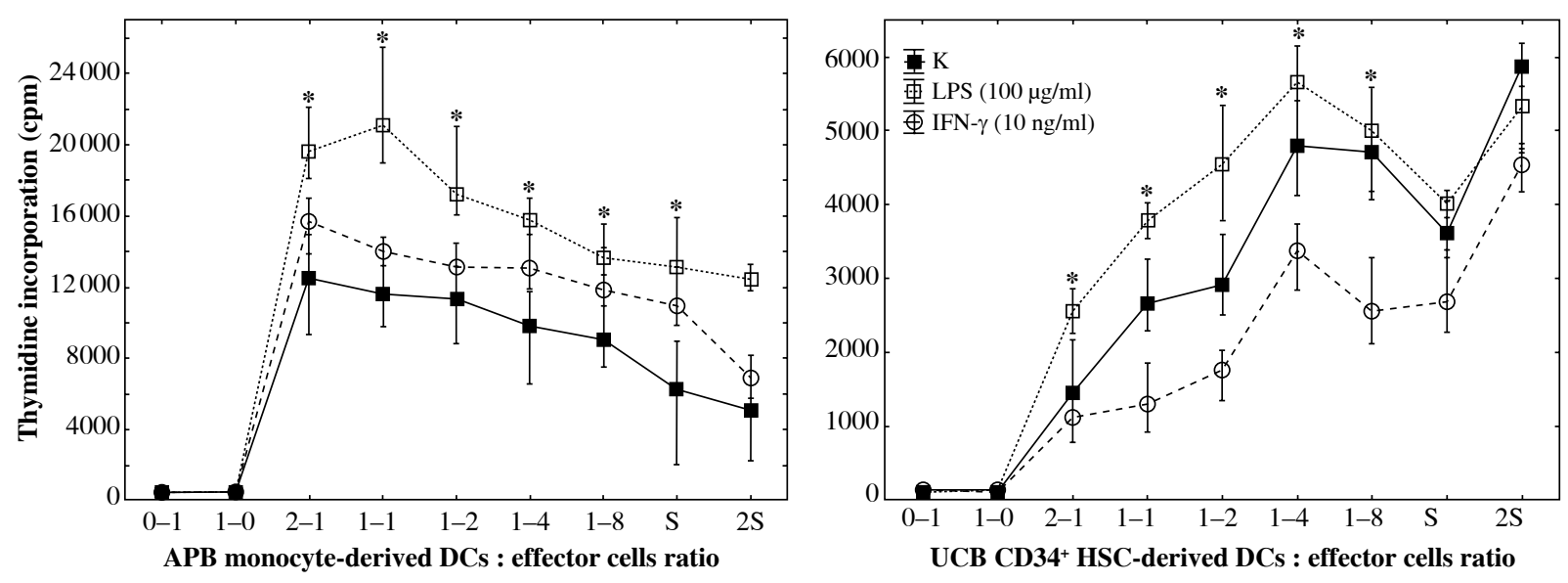

Fig. 5. DCs derived from different types of precursors were shown to exert opposite effects on effector cells in MLC after the challenge with anti-CD3/CD28 beads. Responders were effector cells rich in T-lymphocytes isolated either from peripheral blood or from cord blood. DCs before culture were stimulated for $24 \mathrm{~h}$ with either LPS (100 $\mu \mathrm{g} / \mathrm{ml})(\square)$ or IFN- $\gamma(10 \mathrm{ng} / \mathrm{ml})(\mathrm{O})$. DCs were mixed with effectors in the following ratios $-1: 0$ and $0: 1$ (controls), $2: 1,1: 1,1: 2$, $1: 4$, and $1: 8$. The controls $\mathrm{S}$ and $2 \mathrm{~S}$ contained effectors stimulated with either single or double doses of anti-CD3/ CD28 beads. The results are presented as medians and minimum-maximum $\left(^{*}-\right.$ statistical significance, ANOVA Kruskal-Wallis test, $p<0.05$ )

tration of LPS, the stronger the observed proliferation of effectors in MLC (Fig. 5). UCB All-DCs after LPS stimulation exhibited stronger capacity to stimulate effectors during MLC than the control and IFN- $\gamma$-stimulated cells.

\section{Discussion}

In the last decade, DC-based immunotherapy has been expected to become a new therapeutic tool for cancers and infectious diseases $[3,4,9,18]$. The critical issues underlying DC-immunotherapy is the limited number of DCs available from each patient and, additionally, the fact that those DCs can represent variable activities: antigen-presenting, cytotoxic, or suppressive [9, 17-19].

The increasing number of novel findings concerning regulation of DC maturation has provoked many new questions and doubts, thus many other analyses must be conducted to obtain reliable answers. The effects of expansion depend on so many factors including the selection of precursor source, maturation, and stimulatory factors; therefore, the more facts concerning DC expansion and features are known the better. This issue is crucial because of its potential clinical application.

Our findings revealed the significance of precursor type for the final expansion effect. For our study we obtained DCs from their APB monocytic precursors to create the standard comparative model for evaluation of UCB precursor-derived DCs. We have established and optimised the expansion methods based on the cytokine cocktail containing GM-CSF and IL-4. UCB precursors were additionally supported by SCF. After stimulation with either LPS or IFN- $\gamma$, the morphology, phenotype, and stimulatory properties of DC subpopulations were analysed.

Our observations demonstrated that DCs generated from APB and UCB precursors were not equivalent and exhibited opposite features when culture in comparable conditions, which seems to be concomitant with previously published results [8, 20-23]. Moreover, we selected two subpopulations of UCB precursors with different adhering properties (adherent [A] and non-adherent [NA] cells). Surprisingly, their analysis provided evidence that they may represent distinct populations. DCs obtained from A and NA precursor types exhibited contrasting features, which, additionally, were shown to differ from All-DCs. These observations are likely to be innovative and worth further exploration. Because of the limited number of UCB $A$ and NA precursors the whole panel of tests could not be conducted, but only the comparison of their morphology and phenotype.

NA-DCs were sensitive to suppressive signals (decreased level of $\mathrm{CD} 80^{+} \mathrm{HLA}-\mathrm{DR}^{+}$cells) but, at the same time, were able to opposite reactions in stimulatory environment (increased level CD80+HLA-DR ${ }^{+}$DCs). However, A-DCs answered both inhibitory (IFN- $\gamma$ ) and stimulatory (LPS) factors in the same way due to a decreasing proportion of $\mathrm{CD} 80^{+} \mathrm{HLADR}^{+} \mathrm{DCs}$. All of these observations seem to confirm functional dissimilarities of all three subpopulations of UCB-derived DCs. Many questions appeared concerning the factors that influenced these cells when culturing together or separated. It can be direct-contact-dependant or the properties of one population could dominate over the other, or even the external signals may 
represent the most critical factor. Many further investigations are necessary to explore this issue to describe exhaustively the relations between cells during in vitro culture. To the best of our knowledge, this is the first comparison of variable precursors originating from UCB.

Our findings suggest that adherent and non-adherent precursors can give different DC subpopulations carrying out opposite functions, which, by extension, would influence the success rate of potential therapy. The practical implication of our discovery is not known, but we hope we will have a chance to explore that issue in the future.

The idea to compare these distinct populations appeared after we found that another groups selected precursors according to their adhering properties [24] and phenotype. Rejecting one of these populations suggested the diversity of cells [25, 26]. Arrighi et al. [25] cultured cells CD34-CD14- and ${ }^{-} D 34^{-} \mathrm{CD} 14^{+}$and Caux $\mathrm{C}$ et al. [26] described distinct features of DCs generated from $\mathrm{CD} 14^{+} \mathrm{CD} 1 \mathrm{a}^{-}$vs. CD14-CD1a ${ }^{+}$. However, there is a lack of publications concerning DCs obtained from morphologically different UCB precursors.

In our study DCs generated from precursors of different origin had distinct potency in MLC reaction, which may account for the potential development of anti-cancer vaccines. UCB DCs inhibited proliferation of effectors during MLC test. LPS-stimulated cells achieved stronger stimulatory properties in comparison to unstimulated control and IFN- $\gamma$ stimulated cells. DCs derived from APB monocytes significantly increased their stimulatory properties after incubation with either LPS or IFN- $\gamma$.

When we analysed the results of other groups, we found that some of their observations were concomitant with ours, and some were totally different. In some studies, CD40L enhanced monocyte-derived DC reactivity in MLC tests [20]. The analysis of DCs derived from UCB or APB plastic adherent cells showed that stimulatory properties depend on many factors, like cytokine cocktail used for expansion [8, 21]. Bracho et al. [8], despite similar expression of co-stimulatory and DC markers, demonstrated a significantly reduced alloreactivity of DC derived from plastic-adherent MNC from UCB compared with APB. At the same time, Zheng et al. [21] showed equivalent alloreactivity of UCB and APB - MNC-derived DCs. Additionally, other groups showed that $\mathrm{CD} 34^{+}-$and monocyte-derived DCs are not functionally equivalent with regard to activation of antigen-specific CTLs [16, 22, 23]. Summing up these reports it seems that the precursors' origin from a suppressive niche, such as UCB, does not have to limit the developmental options. The final effect depends instead on the supplementation of culture medium.

In vivo the various anatomical locations provide a different microenvironment, which affects the DC generation. Thus, DC heterogeneity with respect to phenotype, morphology, and functions is determined by the different cellular origin and by the conditions in the particular tis- sue/organ niche supporting the development and terminal differentiation of these cells. Our findings highlight the significance of precursor origin for features and functions of their progeny; however, as we mentioned before, it is not the only issue important during expansion. UCB seems to be a niche that gives rise to immunosuppressive cells even in a stimulatory environment. This knowledge would help to determine the optimal source for generation of DCs performing specific features, which would influence the immune system in a desired manner, safe for the patient, and would enable cellular transplants with much greater probability of success.

Much attention should be paid to optimising the methodology for the culturing of different subtypes of DCs, including the choice of precursor source and usage of specific modulators of their generation and maturation. Our study sheds new light on the cultured and manipulated in vitro DC transplantation issue. The aspects mentioned in our paper require further investigation, and at the same time we have shown that the expansion and differentiation of immunological cells is still a mysterious field.

\section{Acknowledgements}

We would like to thank the volunteers and donors of UCB at each of the locations previously specified. We are grateful for the efforts of the attending physicians and the staff from the Obstetric-Gynaecological Department of St. Adalbert Hospital in Gdansk and from the Department of Obstetrics of the Medical University of Gdansk.

Our study was supported by Polish State Committee for Scientific Research (Grant no. 2 PO5A 088 30).

The authors declare no conflict of interest.

\section{References}

1. Han S, Wang Y, Wang B, et al. (2010): Ex vivo development, expansion and in vivo analysis of a novel lineage of dendritic cells from hematopoietic stem cells. J Immune Based Ther Vaccines 8: 8.

2. Chiang CL, Maier DA, Kandalaft LE, et al. (2011): Optimizing parameters for clinical-scale production of high IL-12 secreting dendritic cells pulsed with oxidized whole tumor cell lysate. J Transl Med 9: 198.

3. Syme R, Bajwa R, Robertson L, et al. (2005): Comparison of CD34 and monocyte-derived dendritic cells from mobilized peripheral blood from cancer patients. Stem Cells 23: 74-81.

4. Harada Y, Okada-Nakanishi Y, Ueda Y, et al. (2011): Cytokine-based high log-scale expansion of functional human dendritic cells from cord-blood CD34-positive cells. Sci Rep 1: 174.

5. Balan S, Kale VP, Limaye LS (2009): A simple two-step culture system for the large-scale generation of mature and functional dendritic cells from umbilical cord blood CD34+ cells. Transfusion 49: 2109-2121. 
6. Li Y, Liu M, Yang ST (2014): Dendritic cells derived from pluripotent stem cells: Potential of large scale production. World J Stem Cells 6: 1-10.

7. Lakomy D, Janikashvili N, Fraszczak J, et al. (2011): Cytotoxic dendritic cells generated from cancer patients. J Immunol 187: 2775-2782.

8. Bracho F, van de Ven C, Areman E, et al. (2003): A comparison of ex vivo expanded DCs derived from cord blood and mobilized adult peripheral blood plastic-adherent mononuclear cells: decreased alloreactivity of cord blood DCs. Cytotherapy $5: 349-361$.

9. Shi J, Ikeda K, Fujii N, et al. (2005): Activated human umbilical cord blood dendritic cells kill tumor cells without damaging normal hematological progenitor cells. Cancer Sci 96: 127-133.

10. Xu RL, Tang Y, Ogburn PL, et al. (2004): Implication of delayed TNF-alpha exposure on dendritic cell maturation and expansion from cryopreserved cord blood CD34+ hematopoietic progenitors. J Immunol Methods 293:169-182.

11. Ballestrero A, Boy D, Moran E, et al. (2008): Immunotherapy with dendritic cells for cancer. Adv Drug Deliv Rev 60: 173-183.

12. Palucka K, Ueno H, Fay J, Banchereau J (2011): Dendritic cells and immunity against cancer. J Intern Med 269: 64-73.

13. Broxmeyer HE, Srour EF, Hangoc G, et al. (2003): High-efficiency recovery of functional hematopoietic progenitor and stem cells from human cord blood cryopreserved for 15 years. Proc Natl Acad Sci U S A 100: 645-650.

14. Kurtzberg J. (2009): Update on umbilical cord blood transplantation. Curr Opin Pediatr 21: 22-29.

15. Kurtzberg J, Prasad VK, Carter SL, et al. (2008): Results of the Cord Blood Transplantation Study (COBLT): clinical outcomes of unrelated donor umbilical cord blood transplantation in pediatric patients with hematologic malignancies. Blood 112: 4318-4327.

16. Ferlazzo G, Wesa A, Wei WZ, Galy A. (1999): Dendritic cells generated either from $\mathrm{CD} 34+$ progenitor cells or from monocytes differ in their ability to activate antigen-specific CD8+ T cells. J Immunol 163: 3597-3604.

17. Liu EM, Law HK, Lau YL (2010): Mycobacterium bovis bacillus Calmette-Guerin treated human cord blood monocyte-derived dendritic cells polarize naive $\mathrm{T}$ cells into a tolerogenic phenotype in newborns. World J Pediatr 6: 132140.

18. Mellor AL, Baban B, Chandler P, et al. (2003): Cutting edge: induced indoleamine 2,3 dioxygenase expression in dendritic cell subsets suppresses T cell clonal expansion. J Immunol 171: 1652-1655.

19. Miller G, Pillarisetty VG, Shah AB, et al. (2003): Murine Flt3 ligand expands distinct dendritic cells with both tolerogenic and immunogenic properties. J Immunol 170: 3554-3564.

20. Chen B, Stiff P, Sloan G, et al. (2001): Replicative response, immunophenotype, and functional activity of monocyte-derived versus CD34(+)-derived dendritic cells following exposure to various expansion and maturational stimuli. Clin Immunol 98: 280-292.

21. Zheng Z, Takahashi M, Narita M, et al. (2000): Generation of dendritic cells from adherent cells of cord blood by culture with granulocyte-macrophage colony-stimulating factor, interleukin-4, and tumor necrosis factor-alpha. J Hematother Stem Cell Res 9: 453-464.
22. Mortarini R, Anichini A, Di Nicola M, et al. (1997): Autologous dendritic cells derived from CD34+ progenitors and from monocytes are not functionally equivalent antigen-presenting cells in the induction of melan-A/Mart-1(27-35)-specific CTLs from peripheral blood lymphocytes of melanoma patients with low frequency of CTL precursors. Cancer Res 57: 5534-5541.

23. Liu S, Yu Y, Zhang M, et al. (2001): The involvement of TNF-alpha-related apoptosis-inducing ligand in the enhanced cytotoxicity of IFN-beta-stimulated human dendritic cells to tumor cells. J Immunol 166: 5407-5415.

24. Chapoval AI, Tamada K, Chen L (2000): In vitro growth inhibition of a broad spectrum of tumor cell lines by activated human dendritic cells. Blood 95: 2346-2351.

25. Arrighi JF, Hauser C, Chapuis B, et al. (1999): Long-term culture of human CD34(+) progenitors with FLT3-ligand, thrombopoietin, and stem cell factor induces extensive amplification of a CD34(-)CD14(-) and a CD34(-)CD14(+) dendritic cell precursor. Blood 93: 2244-2252.

26. Caux C, Massacrier C, Vanbervliet B, et al. (1997): CD34+ hematopoietic progenitors from human cord blood differentiate along two independent dendritic cell pathways in response to granulocyte-macrophage colony-stimulating factor plus tumor necrosis factor alpha: II. Functional analysis. Blood 90: $1458-1470$. 